\title{
BAYES-LOSVD: A Bayesian framework for non-parametric extraction of the line-of-sight velocity distribution of galaxies ${ }^{\star}$
}

\author{
J. Falcón-Barroso ${ }^{1,2}$ and M. Martig ${ }^{3}$
}

\author{
${ }^{1}$ Instituto de Astrofísica de Canarias, Vía Láctea s/n, 38205 La Laguna, Tenerife, Spain \\ e-mail: jfalcon@iac.es \\ 2 Departamento de Astrofísica, Universidad de La Laguna, 38200 La Laguna, Tenerife, Spain \\ 3 Astrophysics Research Institute, Liverpool John Moores University, 146 Brownlow Hill, Liverpool L3 5RF, UK \\ e-mail: M.Martig@ljmu.ac.uk
}

Received 9 October 2020 / Accepted 23 November 2020

\begin{abstract}
We introduce BAYES-LOSVD, a novel implementation of the non-parametric extraction of line-of-sight velocity distributions (LOSVDs) in galaxies. We employed Bayesian inference to obtain robust LOSVDs and associated uncertainties. Our method relies on a principal component analysis to reduce the dimensionality on the set of templates required for the extraction and thus increase the performance of the code. In addition, we implemented several options to regularise the output solutions. Our tests, conducted on mock spectra, confirm the ability of our approach to model a wide range of LOSVD shapes, overcoming limitations of the most widely used parametric methods (e.g., Gauss-Hermite expansion). We present examples of LOSVD extractions for real galaxies with known peculiar LOSVD shapes, including NGC 4371, IC 0719, and NGC 4550, using MUSE and SAURON integral-field unit (IFU) data. Our implementation can also handle data from other popular IFU surveys (e.g., ATLAS ${ }^{3 \mathrm{D}}$, CALIFA, MaNGA, SAMI).
\end{abstract}

Key words. methods: data analysis - techniques: spectroscopic - galaxies: general - galaxies: kinematics and dynamics galaxies: elliptical and lenticular, cD - galaxies: spiral

\section{Introduction}

Galaxies are made up of stars that move on orbits with different degrees of coherence around their nuclei. Each orbital group contains detailed information about the assembly history of each component and thus retains some memory of the different accretion events suffered by the galaxy over time. This information is encoded in their line-of-sight velocity distribution (LOSVDs), and thus by extracting this property we have access to vital clues to unravel the formation and evolution of galaxies we see today. The analysis of the LOSVD can be done directly in the Milky Way and in the local Universe by tracing the motions of stars with common stellar populations along a given line of sight (e.g., Norris 1986; Tolstoy et al. 2004; Deason et al. 2011; Kunder et al. 2012; Ness et al. 2013; Debattista et al. 2015; Zoccali et al. 2017; Du et al. 2020). This is a much more difficult task in nearby galaxies beyond the Local Group, where stars are unresolved and thus the LOSVD at a given position in a galaxy represents multiple populations along that line of sight.

The extraction of the LOSVD of galaxies has been an active field of research for many decades. Both parametric and nonparametric approaches have been developed over the years to address this issue. Interestingly, original implementations prioritised non-parametric over parametric recoveries, something that has radically changed in the last few decades. The most popular approaches include: Fourier correlation quotient (FCQ; Simkin 1974; Sargent et al. 1977; Franx \& Illingworth 1988;

* Details of the code and relevant documentation are freely available in the dedicated repository: https://github.com/ jfalconbarroso/BAYES-LOSVD
Bender 1990); cross-correlation (XC or CCF; Tonry \& Davis 1979; Statler 1995); maximum penalised likelihood (MPL; Saha \& Williams 1994; Merritt 1997; Pinkney et al. 2003); and direct fitting in pixel space (e.g., Rix et al. 1992; Kuijken \& Merrifield 1993; van der Marel \& Franx 1993; Gebhardt et al. 2000; Kelson et al. 2000; Cappellari \& Emsellem 2004; Ocvirk et al. 2006; Chilingarian et al. 2007).

The extraction of the LOSVD is a degenerate problem, as there are an indefinite number of combinations of stellar populations and LOSVD shapes that can explain a particular spectroscopic observation. Breaking the degeneracies implies perfect knowledge of the underlying stellar populations that contribute to a particular line of sight. In the last few decades, this issue has been mitigated with the advent of a large number of intermediate-resolution stellar libraries and stellar population models (see e.g., Bruzual \& Charlot 2003; Valdes et al. 2004; Coelho et al. 2005; Sánchez-Blázquez et al. 2006; Prugniel et al. 2007; Vazdekis et al. 2010; Gonneau et al. 2020; Maraston et al. 2020) that have helped to greatly reduce the so-called effect of template mismatch (e.g., Falcón-Barroso et al. 2003). Another important aspect is the uncertainty of the recovered LOSVD. The methods cited above handle this in different ways. Most of them approach it using Monte Carlo simulations to perturb the input spectrum several times with some known observed uncertainty (however, see de Bruyne et al. 2003 for a more realistic approach). While in recent years the use of parametric forms of the LOSVD has prevailed in the literature (e.g., Emsellem et al. 2011; Falcón-Barroso et al. 2017; van de Sande et al. 2017), it is becoming increasingly clear that non-parametric approaches are needed to describe the complexity in the LOSVD shapes observed in real data (e.g., Jore et al. 1996; Kuijken et al. 1996; 
Halliday et al. 2001; González-García et al. 2006; Katkov et al. 2011; Coccato et al. 2013; Fabricius et al. 2014; Pizzella et al. 2018) and numerical simulations (e.g., Jesseit et al. 2007; Martig et al. 2014; Schulze et al. 2017).

Bayesian inference methods (e.g., Hoffman \& Gelman 2014) offer a natural way of treating both: (1) the uncertainties in the fitting process, with the advantage that they allow the inclusion of our knowledge of the problem during the fitting process via priors on the input parameters; and (2) the true, complex, non-parametric nature of the LOSVDs. Saha \& Williams (1994, hereafter SW94) already considered this particular way of framing the problem, but limitations in computer performance did not allow them to perform a fully general optimisation of both the LOSVDs and templates. In this paper, we revise the SW94 approach and use the latest developments on Bayesian inference and dimensionality reduction techniques to develop a Python ${ }^{1}$ implementation that can efficiently handle template optimisation and robust LOSVD extraction simultaneously.

The paper is organised as follows. We describe the problem of LOSVD extraction, the techniques for dimensionality reduction of the templates and LOSVD regularisation in Sect. 2. We present our tests on mock spectra in Sect. 3 and apply our extraction methods to real data in Sect. 5. We provide all the necessary technical details of our python implementation in Sect. 4, and provide a summary of the paper and outlook for future applications of this methodology in Sect. 6.

\section{The LOSVD extraction}

A LOSVD represents the distribution of the number of stars as a function of velocity along a particular line of sight in a galaxy. In spectroscopic data of nearby galaxies, the LOSVD is the broadening function to be applied to the spectrum of the underlying stellar populations. In essence, the extraction of the LOSVD is a deconvolution problem.

\subsection{The equation}

In its simplest form, the equation that describes the model to be fit to the data can be expressed in mathematical terms as:

$G_{\text {model }}(\lambda)=\sum_{k=1}^{K}\left[w_{k} \cdot T_{k}(\lambda)\right] \star B+C(n)$,

where $w_{k}$ are the weights for each stellar population template $\left(T_{k}\right), B$ is the broadening function (i.e. the LOSVD), the $\star$ operator is a convolution, and $C(n)$ is an additive polynomial of order $n$. The polynomial term is convenient to reduce the impact of template mismatch (which can happen even with the most complete template libraries) and other imperfections during data reduction (e.g., non-perfect sky subtraction and/or scattered light). The equation can become more complicated if the effects of dust attenuation and/or calibration issues between data and templates are to be taken into account (see Eq. (11) in Cappellari 2017 for a more complete example). The implementation presented in this paper uses the prescription shown in Eq. (1), as we have checked that it works for a wide range of datasets, but it can be easily extended to include other correction terms if required.

\footnotetext{
1 https://www .python.org/
}

The optimisation procedure involves the minimisation of the residuals:

$r_{\mathrm{p}}=\frac{G_{\text {data }}\left(x_{\mathrm{p}}\right)-G_{\text {model }}\left(x_{\mathrm{p}}\right)}{\Delta G_{\text {data }}\left(x_{\mathrm{p}}\right)}$,

where $x_{\mathrm{p}}$ is the value of the data or model at a given pixel, $G_{\text {data }}$ is the observed spectrum, $\Delta G_{\text {data }}$ are the observed uncertainties, and $G_{\text {model }}$ is the model presented in Eq. (1). The most commonly used method for the minimisation of Eq. (2) is a least squares one, as there are plenty of computer implementations (e.g., Lawson \& Hanson 1974; Moré et al. 2001; Jones et al. 2001) to perform the fits very efficiently.

Inspired by the work of SW94, we opted for revisiting their Bayesian non-parametric approach for the LOSVD extraction. There were three main aspects of SW94 that were not possible to explore given the computer capabilities and/or mathematical methods available at the time: (1) the Markov chain Monte Carlo (MCMC) sampling strategy; (2) template optimisation; and (3) different forms of regularisation for the LOSVD. We describe the new improvements in the following sections.

\subsection{Markov chain Monte Carlo sampling}

There are multiple possible strategies for the sampling of parameter space in Bayesian inference frameworks. Classical approaches include Metropolis-Hastings (Metropolis et al. 1953; Hastings 1970) or Gibbs (Geman \& Geman 1984) samplers. With more complex models, the field has experienced a spur of new methods to efficiently probe large parameter spaces including nested sampling (e.g., Buchner 2016), Hamiltonian Monte Carlo (e.g., Duane et al. 1987), or Stein Variational Gradient Descent (e.g., Liu \& Wang 2016) to cite a few. We refer the interested reader to Chi Feng's Github webpage ${ }^{2}$ for a demo on the performance of different samplers.

The SW94 approach relied on the Metropolis algorithm for the exploration of parameter space. Our implementation is based on the No-U-Turn-Sampler (NUTS) introduced by Hoffman \& Gelman (2014), that has proven to be a much more effective sampler. This is part of the $\operatorname{Stan}^{3}$ package (Carpenter et al. 2017), which is our software of choice for the MCMC sampling. Stan is a probabilistic programming language for statistical modelling and data analysis used in many fields, from physics or engineering to social sciences. We refer the interested reader to Stenning et al. (2016), Asensio Ramos et al. (2017), Parviainen (2018), Lamperti et al. (2019), and Dullo et al. (2020) for a few examples of Stan applications in astronomy.

Besides the sampling scheme, one of the main advantages of our implementation within the Stan framework is the use of the simplex (i.e. a vector of positive values whose sum is equal to one) to describe the LOSVD. This type of parametrisation provides, naturally, physical and normalisation constraints, and it allows for a very efficient exploration of parameter space during minimisation (Betancourt 2012).

\subsection{Template optimisation}

A crucial element in the recovery of the LOSVD is the basis of stellar templates used to fit the observed spectra. Ideally, such a basis should contain stellar spectra covering the widest possible range of stellar parameters (i.e. $T_{\text {eff }},[\mathrm{Fe} / \mathrm{H}], \log (g)$, and stellar

\footnotetext{
2 http://chi-feng.github.io/mcmc-demo/
}

3 https://mc-stan.org/ 


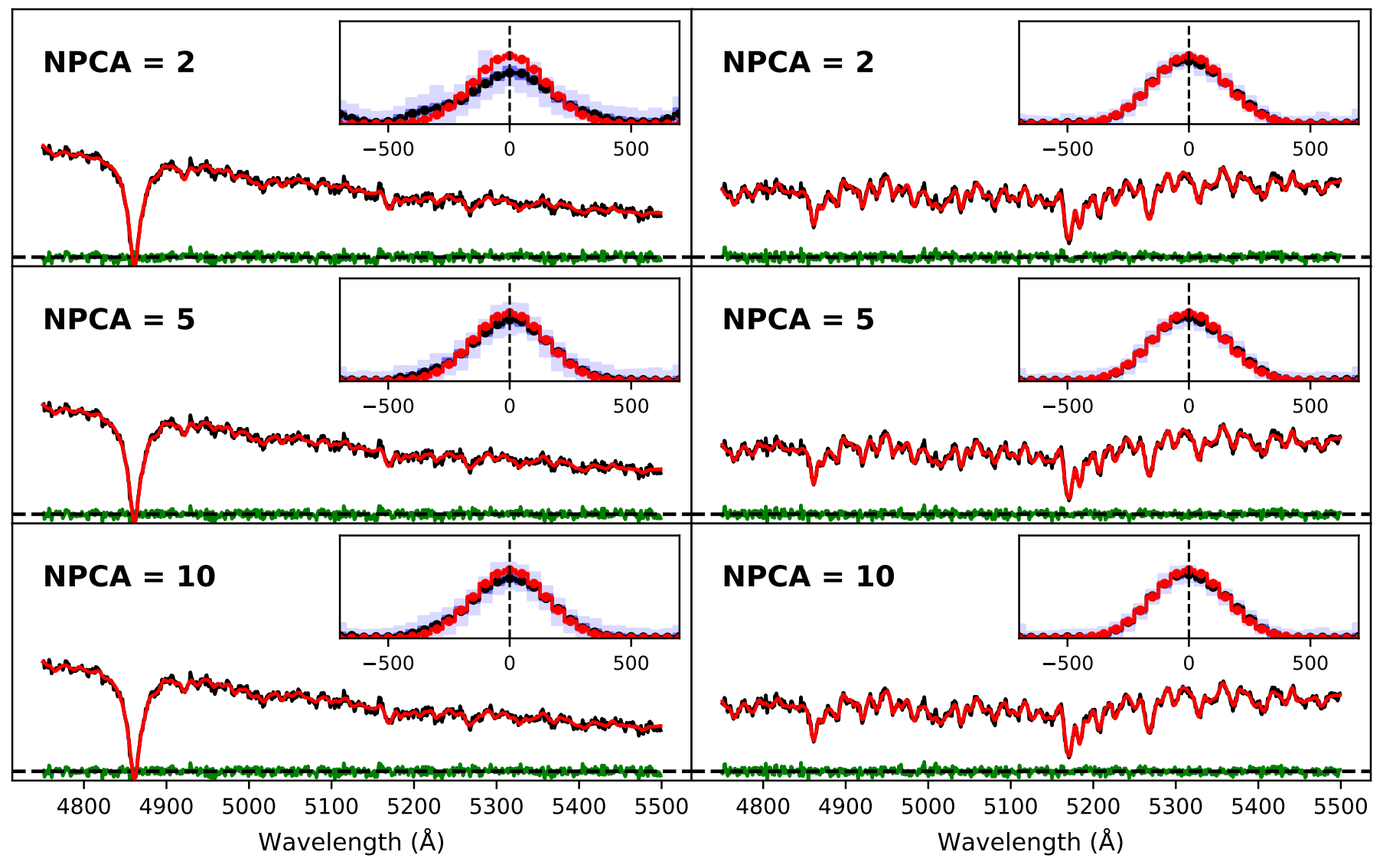

Fig. 1. Comparison of spectral fitting quality and LOSVD recovery for different numbers of PCA components. Each column presents input spectra with different stellar populations (young and old on the left and the right respectively). The input spectra have a S/N of 50 per pixel. All panels are plotted on the same scale. The black lines on the main panels are the input test data, while the red line shows the best fitting model. Residuals are indicated in green. The spectral fits are carried out with 2, 5, and 10 PCA templates (from top to bottom as indicated). In the insets, the input LOSVD is a Gaussian centred at zero and a velocity dispersion of $150 \mathrm{~km} \mathrm{~s}^{-1}$ (indicated in red). Units of the abscissae in the insets are km s${ }^{-1}$. The recovered median values of the LOSVDs are indicated with a thick black line. 16\%-84\% and 1\%-99\% confidence limits at each point are indicated in dark and light blue, respectively. An order 2 auto-regressive prior was used to perform the fitting (see Sect. 2.4 for details).

abundances) or stellar population models with a good sampling of, for example, ages, metallicities, IMF shapes and slopes, and possibly different chemical abundance ratios. As already mentioned in the introduction, this is now possible with the advent of the latest stellar libraries and models.

Using the typical set of $\sim 500-1000$ templates, the minimisation involves finding the weights $\left(w_{k}\right)$ for each of them and performing the convolution of the LOSVD on $\sim 1000$ spectral pixels per fitting iteration. This is a very time-consuming task even for the most efficient Bayesian samplers. Since reducing the number of pixels to fit may not be an option, we are thus left with the only alternative of decreasing the number of templates to be used in the optimisation process. This will have the advantage of not only reducing the number of parameters (i.e. $w_{k}$ ), but also boosting minimisation performance by very large factors (e.g., from days to minutes).

There are a number of well-known techniques for dimensionality reduction that one could use to whittle down the number of templates: for example, independent component analysis (Lu et al. 2006), factor analysis (Nolan et al. 2006), non-negative matrix factorisation (Blanton \& Roweis 2007), diffusion maps (Richards et al. 2009), or $K$-means (Sánchez Almeida \& Allende Prieto 2013). Among all alternatives, we favoured the well-known principal component analysis (PCA) method. PCA has been extensively used in astrophysics for problems very much related to spectral fitting (e.g., Ronen et al. 1999;
Li et al. 2005; Chen et al. 2012) and has proven to be both robust and very effective in speeding up calculations. With regard to our particular problem, the use of PCA has improved performance from several days to a few minutes (i.e. a 500-fold decrease in computing time).

In practice, the use of PCA means capturing well over $99 \%$ of the variance present in a template library of $\sim 1000$ spectra with fewer than ten PCA components. Figure 1 shows the effect of using different numbers of PCA components during the fit for two mock spectra ${ }^{4}$ with a signal-to-noise ratio per pixel (hereafter $\mathrm{S} / \mathrm{N}$ ) of 50 . It is remarkable how as few as two PCA components can already reproduce the observed spectrum with great accuracy. This is in part due to the use of additive polynomial that helps to reduce potential template mismatch issues. We checked the recovery for different types of stellar populations (as shown in the two columns), and the extracted LOSVD is less accurate for young stellar populations with a low number of PCA components. This is not unexpected given that spectra of younger populations show greater variation, which is difficult to capture with just two PCA components. We noticed that the closer the input spectrum is to the average spectra of the

4 For reference, for this particular case, the continuum-to-line ratio (defined as $100 \times \sigma_{\text {residuals }} / \sigma_{\text {spectrum }}$ ) is $13 \%$ and $23 \%$ for the young and old populations, respectively: almost independent of the number of PCA components used in the fitting procedure. 
templates (i.e. intermediate stellar populations in our particular case), the better the recovery is for low number of PCA components. In general, our tests suggest that five PCA components suffice to recover the input LOSVD with great accuracy, with a clear improvement as $\mathrm{S} / \mathrm{N}$ increases.

The optimal selection of the number of PCA components is not a well defined quantity, however. Choosing a number of PCA components that would explain a certain variance of the spectra (e.g., compatible with the noise of the spectrum) seems a reasonable approach. However, in practice, for real data this means selecting a different number of components for each spectrum of the dataset, which is very impractical. In our experience, for typical $\mathrm{S} / \mathrm{N}$ ratios between 50 and 100 , it is sufficient to choose a number between five and ten PCA components ${ }^{5}$. In our implementation, this number is a free parameter that the user has to decide on before runtime.

\subsection{LOSVD regularisation}

The extraction of the LOSVD is a degenerate problem with a large number of LOSVD shapes and combinations of templates that can reproduce the observed spectrum with great accuracy. Nevertheless, the range of allowed LOSVD solutions can be constrained for reasonably high $\mathrm{S} / \mathrm{N}$ data.

There are different ways to impose some level of regularisation onto the output LOSVD. In our bayesian approach they are expressed in the form of priors over the LOSVD. We explored four different types of priors:

1. No regularisation:

$$
\operatorname{LOSVD}_{i} \sim \mathcal{N}\left(0, \sigma^{2}\right),
$$

where $\operatorname{LOSVD}_{i}$ is the $i$ th velocity element of the LOSVD, and $\mathcal{N}\left(0, \sigma^{2}\right)$ represents a normal distribution with mean zero and variance $\sigma^{2}$. This is a fairly uninformative prior that assumes the same level of uncertainty on all LOSVD elements.

2. Random-walk prior:

$$
\operatorname{LOSVD}_{i} \sim \mathcal{N}\left(\operatorname{LOSVD}_{i-1}, \sigma^{2}\right),
$$

where the $i$ th element of the LOSVD is linked to the previous one. This type of prior was the one proposed by SW94. We set the prior for the first element to $\operatorname{LOSVD}_{0} \sim \mathcal{N}\left(0, \sigma^{2}\right)$.

3. Auto-regressive prior:

$$
\operatorname{LOSVD}_{i} \sim \mathcal{N}\left(\alpha+\sum_{k=1}^{N} \beta_{k} \times \operatorname{LOSVD}_{i-k}, \sigma^{2}\right) .
$$

This is a more general form of prior that becomes equal to the random-walk for $\alpha=0$ and $\beta=1$ for $k=1$. Stronger regularisation is obtained by increasing the order $k$, which links more consecutive LOSVD elements. We imposed the same prior used for the random-walk approach to the first element of the LOSVD, and used weakly informative normal priors for $\alpha$ and $\beta$.

4. Penalised B-splines

B-splines are a special type of piecewise polynomials controlled by knots that are often used for interpolation (e.g., Press et al. 2003). One of the major difficulties on the definition of B-splines is the choice of the number of knots to define the polynomial function. In our Stan implementation,

\footnotetext{
5 This is using the MILES models (Vazdekis et al. 2010).
}

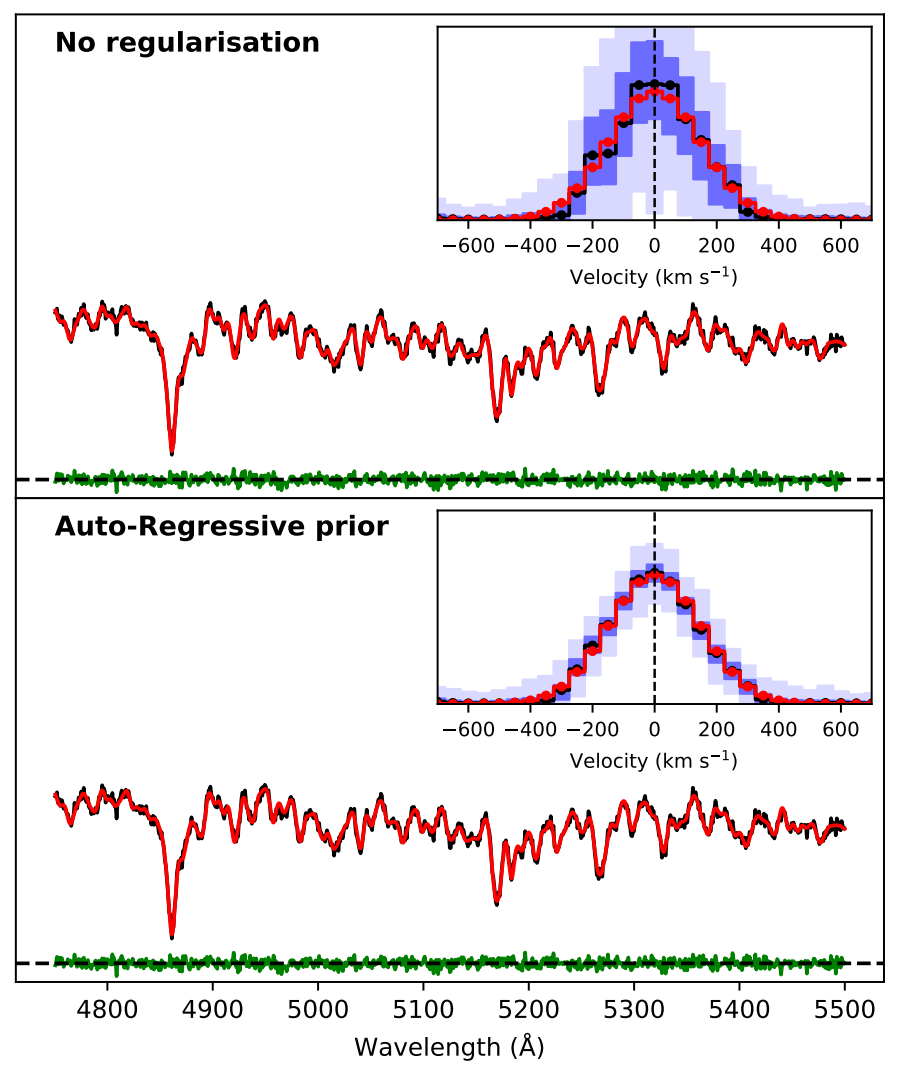

Fig. 2. LOSVD recovery for no regularisation and an auto-regressive (order 2) prior. Colours as in Fig. 1.

we followed the example provided by Milad Kharratzadeh ${ }^{6}$, and rather than establishing a relation between different elements of the LOSVD, we applied random-walk priors to the B-spline coefficients such that

$a_{1} \sim \mathcal{N}(0,1), \quad a_{i} \sim \mathcal{N}\left(a_{i-1}, \tau\right), \quad \tau \sim \mathcal{N}(0,1)$.

This approach promotes smoothness in the resulting LOSVD, preventing excessive wiggling in the solution. Another important parameter that controls the level of flexibility of the B-splines is the B-spline order $k$.

An interesting feature of our procedure is that we do not impose any pre-defined value to $\sigma^{2}$ or $\tau$ during the fitting process. In fact, they are considered nuisance parameters, and we let the quality of the data establish their optimal distributions. Far from being unconstrained, it turns out that both parameters are well behaved and display fairly tight distributions.

We illustrate the effect of the choice of prior for the LOSVD and its uncertainties in Fig. 2. We created a test spectrum for this purpose with a Gaussian LOSVD and a $S / N=100$ per spectral pixel. The figure shows the difference between no regularisation and an auto-regressive prior of order 2. Both approaches deliver an indistinguishable fit to the input spectrum and capture the Gaussian nature of the input LOSVD. However, the level of uncertainty displayed by the case without regularisation is far larger than that of the auto-regressive prior. These results are very much in agreement with the findings of Saha \& Williams (1994). As we show in Sect. 3, this difference persists even at the highest $\mathrm{S} / \mathrm{N}$ levels and is related to the number of degrees of freedom of one method versus the other.

\footnotetext{
6 https://mc-stan.org/users/documentation/ case-studies/splines_in_stan.html
} 


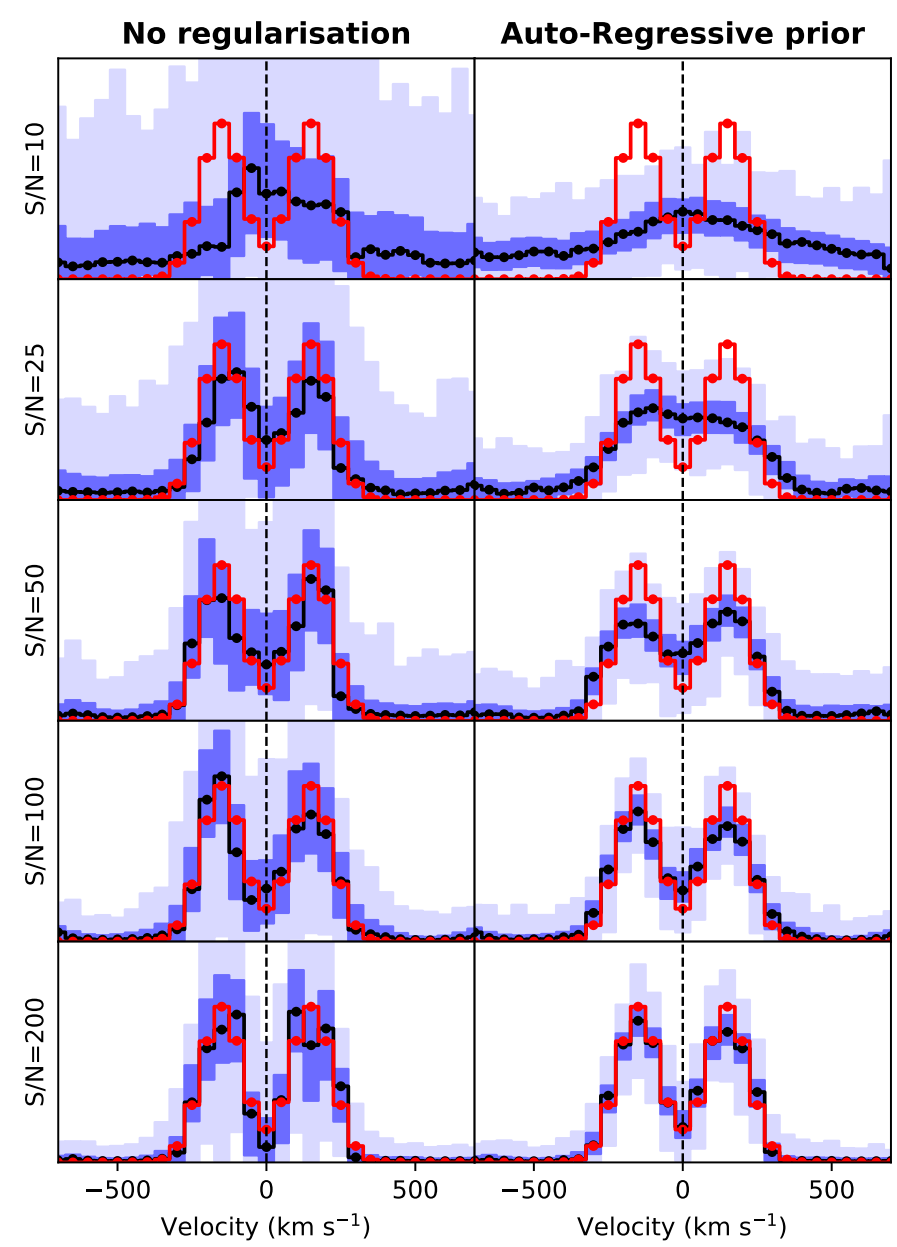

Fig. 3. LOSVD recovery for different $\mathrm{S} / \mathrm{Ns}$ and types of regularisation. We used a double-Gaussian LOSVD profile as an example (indicated in red). Left column: solutions for extraction with no regularisation, right column: results for an order 2 auto-regressive prior. Each row represents two cases for a different $\mathrm{S} / \mathrm{N}$ per pixel as indicated. All panels are plotted on the same scale. Colours as in Fig. 2.

\subsection{Likelihood}

Besides the priors, for the minimisation process, we need to define the form of the likelihood of our data given a model. In our problem, we assume that our spectroscopic observations can be explained by a normal distribution, such that

$G_{\text {data }}(\lambda) \sim \mathcal{N}\left(G_{\text {model }}(\lambda), \sigma_{G_{\text {data }}}^{2}\right)$,

where $G_{\text {data }}(\lambda)$ is the observed input spectrum, $G_{\text {model }}(\lambda)$ is our model, and $\sigma_{G_{\text {data }}}^{2}$ is the variance of the observed spectrum.

Equation (1) is adequate to define our model when a full template library is to be used during the minimisation process. In our case, however, the use of PCA components transforms that equation to the following:

$G_{\text {model }}(\lambda)=\left[\widetilde{T}+\sum_{k=1}^{K} w_{k} \cdot \operatorname{PCA}_{k}(\lambda)\right] \star B+C(n)$,

where $w_{k}$ are now the weights for each PCA component $\left(\mathrm{PCA}_{k}\right)$, $\widetilde{T}$ is the mean template of the input library, $B$ is the broadening function (i.e. the LOSVD), the $\star$ operator is a convolution, and $C(n)$ is an additive polynomial of order $n$. Our implementation in Stan supports both forms of equations, but it is significantly more efficient with Eq. (8).

\section{Tests on simulated data}

We checked the performance of our implementation in different circumstances by creating mock spectra for a wide range of S/Ns and input LOSVD shapes. While some of the LOSVDs were arbitrarily defined by combining Gaussian functions, others come from numerical simulations and are thus more realistic. Here, we choose to illustrate a case with an input spectrum with an intermediate-age stellar population, but results are consistent when other populations were used. Our fits were performed using five PCA components.

Figure 3 shows an example of the recovery of an extreme case of LOSVD shape (i.e. a double-Gaussian profile) for a range of S/Ns and two prior assumptions. The effect of regularisation is evident from the lowest to the highest $\mathrm{S} / \mathrm{Ns}$. With no regularisation, there is a large range of possible solutions, as displayed by the confidence intervals, even at $S / N=200$. It is interesting to note that the level of uncertainty decreases drastically with $\mathrm{S} / \mathrm{N}$ when no regularisation is applied, while the improvement is not so large when priors are used. Regularised solutions come at the price of introducing some bias (see Sect. 5 for an example). This is most acute for the lowest $\mathrm{S} / \mathrm{Ns}$, where the regularised solution fails to capture the double-peaked nature of the input LOSVD. It is clear, however, that at $S / N=10$, even non-regularised solutions cannot reproduce the input LOSVD. It seems that at an $\mathrm{S} / \mathrm{N}$ of 25 , the non-regularised solution already clearly reveals the double-peak feature, while the regularised one does not. This is an important result, as it shows that non-regularised solutions are more accurate at low $\mathrm{S} / \mathrm{N}$ regimes, at the expense of larger uncertainties.

In Fig. 4, we present how the different types of regularisation methods listed in Sect. 2.4 influence the recovery of the range of input LOSVDs we prepared for these tests. The $\mathrm{S} / \mathrm{N}$ of the input data is 50 per spectral pixel. The first thing to notice is that the overall level of accuracy in the LOSVD recovery is rather good (i.e. the input LOSVD is contained within the confidence levels) for all LOSVD shapes. Nevertheless, as expected from previous figures, the use of different priors result in different confidence intervals. An interesting feature is the difference in the confidence intervals between random-walk and auto-regressive (order 1) priors. This is entirely driven by the reduced number of degrees of freedom of the former compared to the latter (see Sect. 2.4). Perhaps one of the most important messages from the figure is that subtle details can be extracted at $S / N=50$. This is particularly striking for the case displayed in the bottom row. This LOSVD is made of two Gaussians: a prominent one centred at $-200 \mathrm{~km} \mathrm{~s}^{-1}$, and a faint component located at $500 \mathrm{~km} \mathrm{~s}^{-1}$. The goal of this test was to check the ability of the code to recover the presence of potential small satellites being accreted onto a galaxy. This appears to have been achieved for any choice of regularisation, with a slightly better recovery of the faint component with the random-walk and auto-regressive priors. These results are quite encouraging, as it means that there is no need for very high $\mathrm{S} / \mathrm{N}$ ratios to detect this kind of feature. We provide versions of Fig. 4 for different S/Ns in Appendix A.

\section{Implementation with pyStan}

There are many alternatives one could choose from to implement all the ideas proposed in Sect. 2. Specifically, in Python some of the most popular packages include emcee ${ }^{7}$ (Foreman-Mackey et al. 2013) and pyMC $3^{8}$ (Salvatier et al. 2016), but we invite the

\footnotetext{
7 https://emcee. readthedocs.io/

8 https://docs.pymc.io/
} 


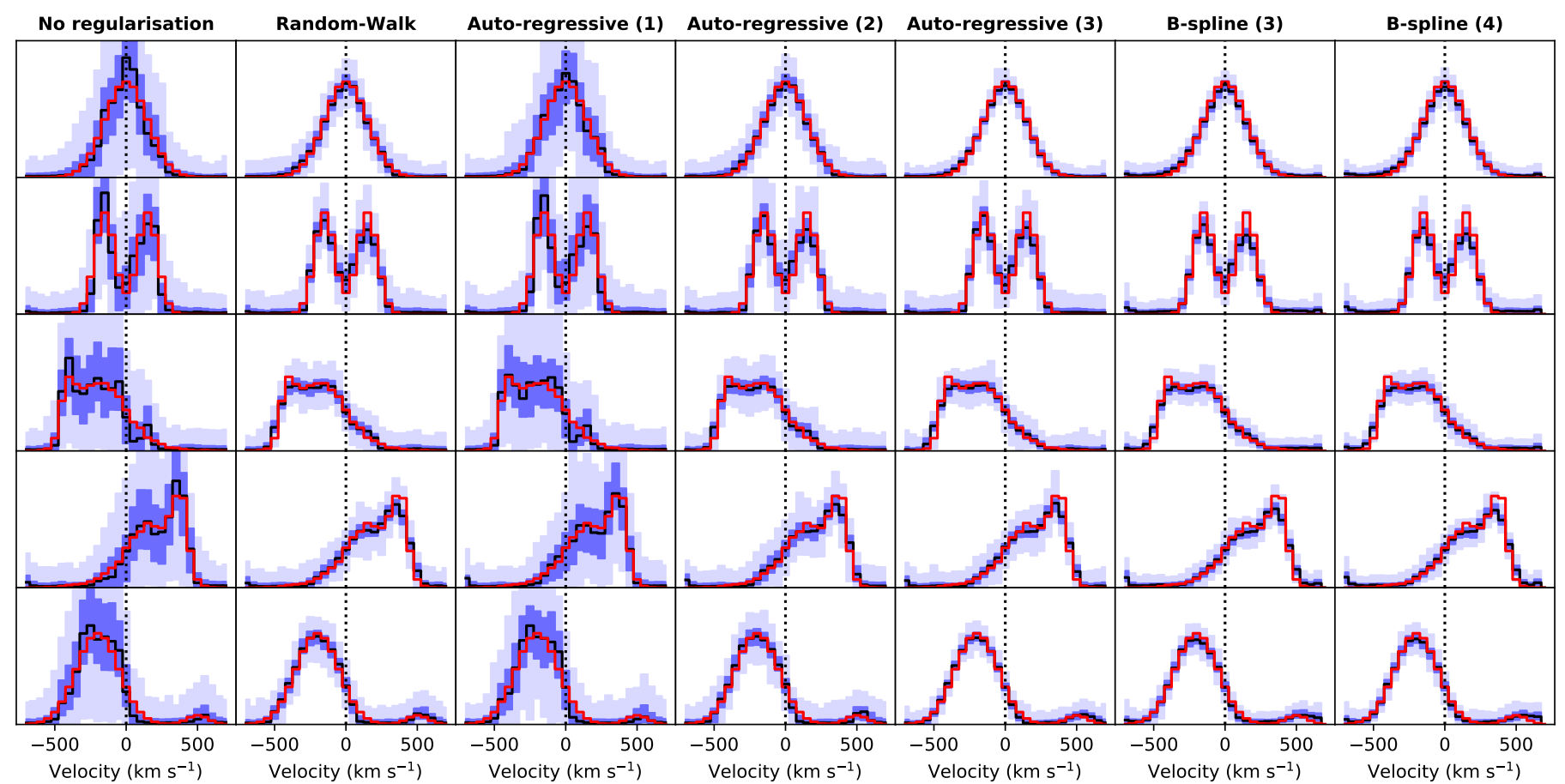

Fig. 4. LOSVD recovery for different input LOSVDs and types of regularisation. Colours as in Fig. 2. Each row represents a particular LOSVD shape for different types of regularisation. These are solutions for input spectra with $S / N=50$ per pixel. All panels are plotted on the same scale.

reader to consult Gabriel Perren's webpage ${ }^{9}$ for a comprehensive list of options. We picked pyStan ${ }^{10}$ as our package of choice to develop BAYES-LOSVD as it offers a convenient python interface to Stan.

BAYES-LOSVD is built in a modular fashion to make it very simple for the end user to extend its capabilities. This includes the addition of (1) read-in routines for data of a new instrument; (2) new Stan models with different kinds of regularisation; and (3) new template libraries. Our current implementation supports datacubes from the MUSE-WFM, SAURON, ATLAS ${ }^{3 \mathrm{D}}$, CALIFA, MaNGA, and SAMI surveys, as well as the possibility to read standard 2D FITS format files with spectra along the rows. The linearly sampled input spectra are pre-processed before execution, allowing the user to pick the level of Voronoi binning (using Cappellari \& Copin 2003 implementation), the velocity range, and sampling of the output LOSVD. In this process, the designated templates will be prepared accordingly, with the possibility of switching off the reduction of the template basis with the PCA scheme described in Sect. 2.3.

Upon execution, the user can decide whether to analyse the entire set of Voronoi bins or a just a selection of them. Distributed computing is implemented natively, so that multiple spectra can be executed in parallel on multi-CPU machines. On output, by default, only summary statistics are stored. MCMC posterior distributions are described with highest density interval estimators (e.g., Kruschke 2014), which are more accurate to describe highly skewed distributions (as opposed to the standard percentiles approach). They are stored on disc in HDF5 format $^{11}$. Diagnostic plots are also created if requested. Users wanting to delve into the details can chose to save the entire posterior distribution values, which can then be easily analysed using the Arviz ${ }^{12}$ package.

Performance and execution times depend very much on the data, and on the parameters used for the LOSVD extraction. Thanks to Stan, convergence is usually achieved with a very small number of iterations. In all the tests presented here, three chains with 500 iterations (i.e. warm-up + sampling) sufficed to obtain well-behaved posterior distributions. A typical spectrum with $\sim 500$ pixels (e.g., 4800-5500 $\AA$ region) and $S / N=50$ per pixel, a velocity range of $\pm 700 \mathrm{~km} \mathrm{~s}^{-1}$ with a sampling of $50 \mathrm{~km} \mathrm{~s}^{-1}$, and five PCA components would require $\sim 10 \mathrm{~min}$ on a cluster with Intel Xeon E5-2630 (v4) CPUs.

There is one major bottleneck in our implementation: convolution is performed in direct space given Stan's current inability to handle complex numbers. This has a very strong impact when wide spectral ranges are to be fitted. Based on discussions on the Stan forum ${ }^{13}$, we are aware that fast Fourier transforms will be possible in the not-so-distant future. Another aspect where we could already optimise performance is the likelihood evaluation. The latest version of Stan has introduced new features that can accelerate this process by large factors by distributing its computation over many CPUs. This is unfortunately not available in the pyStan version (v2.19) we are using, but it will be available with the release of pyStan (v3). Therefore, there is still room for performance improvements in the mid-term. We remain alert and will update the code to keep up with any new developments.

The code can be accessed via a dedicated Github repository ${ }^{14}$. Detailed documentation can be found on the same page.

\footnotetext{
9 https://gabriel-p.github.io/pythonMCMC/

${ }^{10}$ https://pystan.readthedocs.io

11 https://www.h5py.org/
}

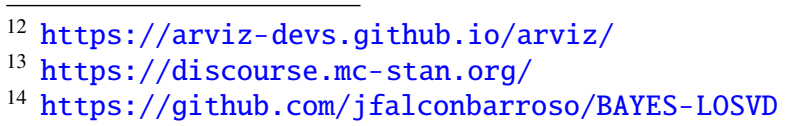



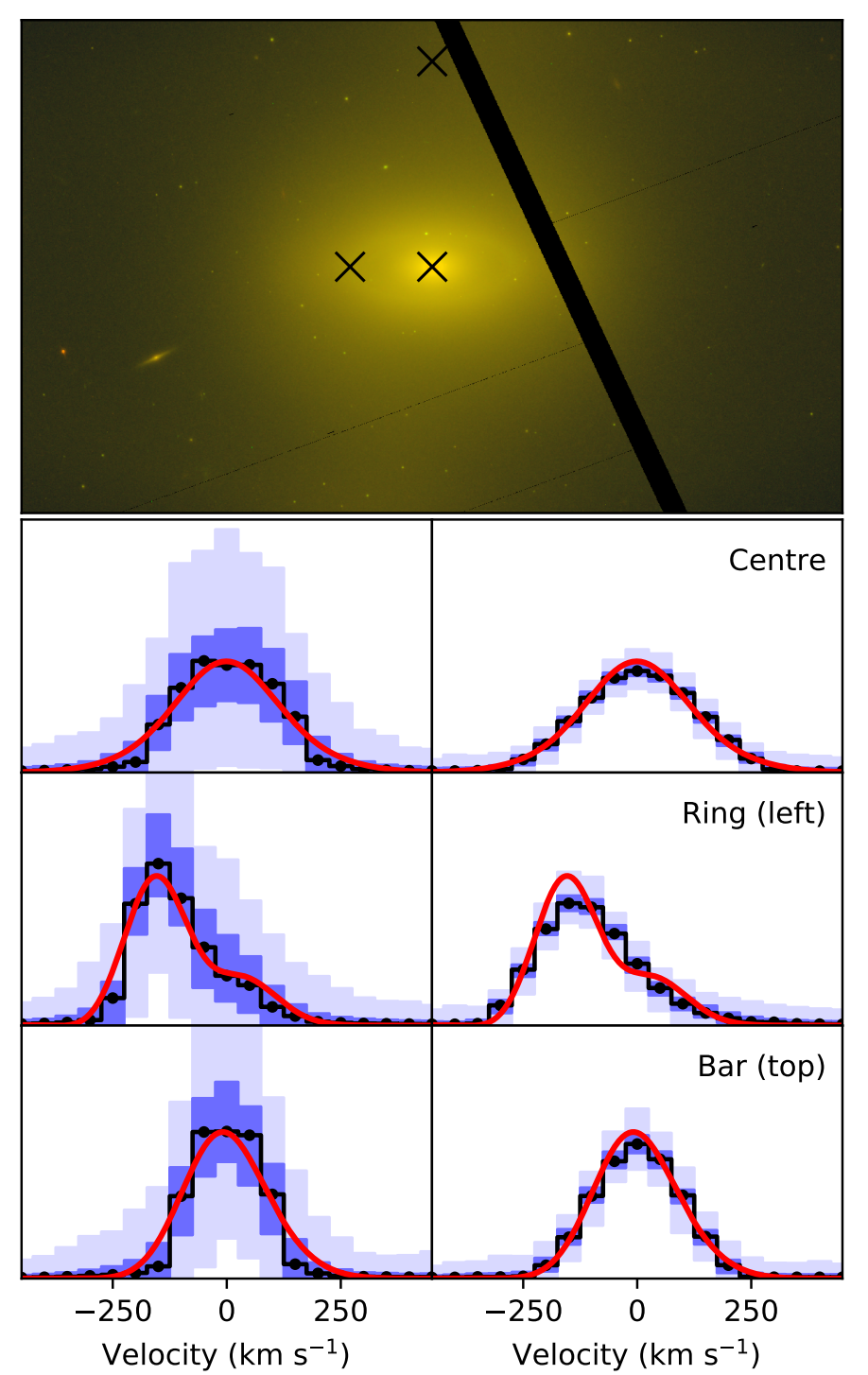

Fig. 5. BAYES-LOSVD extraction for NGC 4371. Top panel: Hubble Space Telescope colour image based on the $F 475 W$ and $F 850 L P$ filters. North is up and east to the left. Bottom panels: LOSVDs extracted at different locations (as indicated with black crosses on the image). On the left, without regularisation, and on the right with an order 2 autoregressive prior. Colours as in Fig. 2. Red lines correspond to the best Gauss-Hermite LOSVD extracted with pPXF (see Sect. 5.1 for details).

\section{Application to real data}

We now turn our attention to the recovery of LOSVD using real data from different instruments. Here, we show examples of LOSVDs for three galaxies presenting LOSVDs with varying degrees of complexity. We chose data from MUSE-WFM and SAURON IFUs, but we note that the code has also been benchmarked with data from some of the most popular IFU surveys (e.g., ATLAS $^{3 \mathrm{D}}$, CALIFA, MaNGA, SAMI).

\subsection{NGC 4371}

The first case we present is NGC 4371. This galaxy was studied in great detail by Gadotti et al. (2015) using the MUSE IFU, and it was part of the pilot programme for the TIMER survey (Gadotti et al. 2019). NGC 4371 is interesting as it is a fairly old system (i.e. $\geq 7$ Gyr throughout) with evidence of a fossil nuclear stellar ring void of star formation (e.g., Erwin et al. 2001). Following the study of Gadotti et al. (2015), we extracted spectra with a 3.0" aperture in three positions of the galaxy: at the centre, the nuclear stellar ring, and a location along the bar. The $\mathrm{S} / \mathrm{N}$ of the spectra in the three apertures is well above 100 per pixel. We imposed a velocity sampling of $50 \mathrm{~km} \mathrm{~s}^{-1}$.

Figure 5 shows the results of our analysis. The three LOSVDs are different from each other in different aspects. While the LOSVDs at the centre and bar regions display fairly Gaussian profiles, the ring-dominated region is clearly asymmetric and skewed. The panels on the left-hand side show solutions with no regularisation, and the ones on the right used an order 2 autoregressive prior. In essence, we see the same behaviour observed with the test data in previous sections with non-regularised solutions giving larger confidence intervals than the regularised ones. The two sets of solutions are very much consistent with each other, as was also observed in our experiments. These results presented in the figure were obtained by applying our method to spectra in the $4800-5300 \AA$ wavelength range. We also computed solutions based on spectra around the calcium triplet region (8450-8700 $\mathrm{A}$ ) and obtained identical solutions (thus not shown here). This was not totally unexpected since there is no evidence of multiple stellar populations in this galaxy.

In addition, for comparison, we plot the best Gauss-Hermite LOSVD extracted with pPXF (Cappellari 2017). In this particular case, the agreement between the recovered LOSVDs with our method and pPXF is very good. This is especially true for the almost Gaussian LOSVDs at the centre and bar locations of the galaxy. At the ring, the regularised solution does not match the pPXF result as closely as the non-regularised one, but differences are still within a $1 \%-99 \%$ percentiles of our non parametric extraction. While for cases like this one, the advantage of the non-parametric approach may not seem evident, it is important to note that the Gauss-Hermite parametrisation allows for negative values on the wings of the LOSVD. This situation occurs for $h_{3}$ and $h_{4}$ values such as 0.1 , and -0.1 respectively, which are not uncommon in the kinematic maps presented in many IFU surveys. Since we constrained the LOSVD during the fit to admit only positive values, our method overcomes this limitation and provides naturally physically meaningful LOSVDs.

\subsection{IC 0719}

The second case we studied was IC 0719, a spectacular case displaying multiple kinematic components (Katkov et al. 2013; Pizzella et al. 2018). This galaxy is made of stars in two counterrotating large-scale discs with distinct stellar populations. In addition, it has an ionised gas component showing the same sense of rotation of the secondary, lower-mass, younger stellar disc. We used MUSE observations around the 4800-5300 $\mathrm{A}$ region to extract three apertures along the major axis of the galaxy. As for the case of NGC 4371, the $\mathrm{S} / \mathrm{N}$ of the spectra is well above 100 per pixel. We imposed a velocity sampling of $50 \mathrm{~km} \mathrm{~s}^{-1}$.

Figure 6 shows an almost perfect Gaussian LOSVD profile at the centre of the galaxy, while LOSVDs along the major axis display clear double-peaked shapes. This is in perfect agreement with the analysis performed by Pizzella et al. (2018). For comparison, we also plot the best-fit Gauss-Hermite LOSVD parametrisation obtained with pPXF (in red). Here, it becomes obvious that the Gauss-Hermite expansion cannot reproduce such complicated shapes well, and it shows LOSVD wings that have slightly negative values. Although not obvious due to the normalisation, for the aperture at $17^{\prime \prime}$ the pPXF extraction creates a third peak in the LOSVD at velocities $\sim 500 \mathrm{~km} \mathrm{~s}^{-1}$, where 

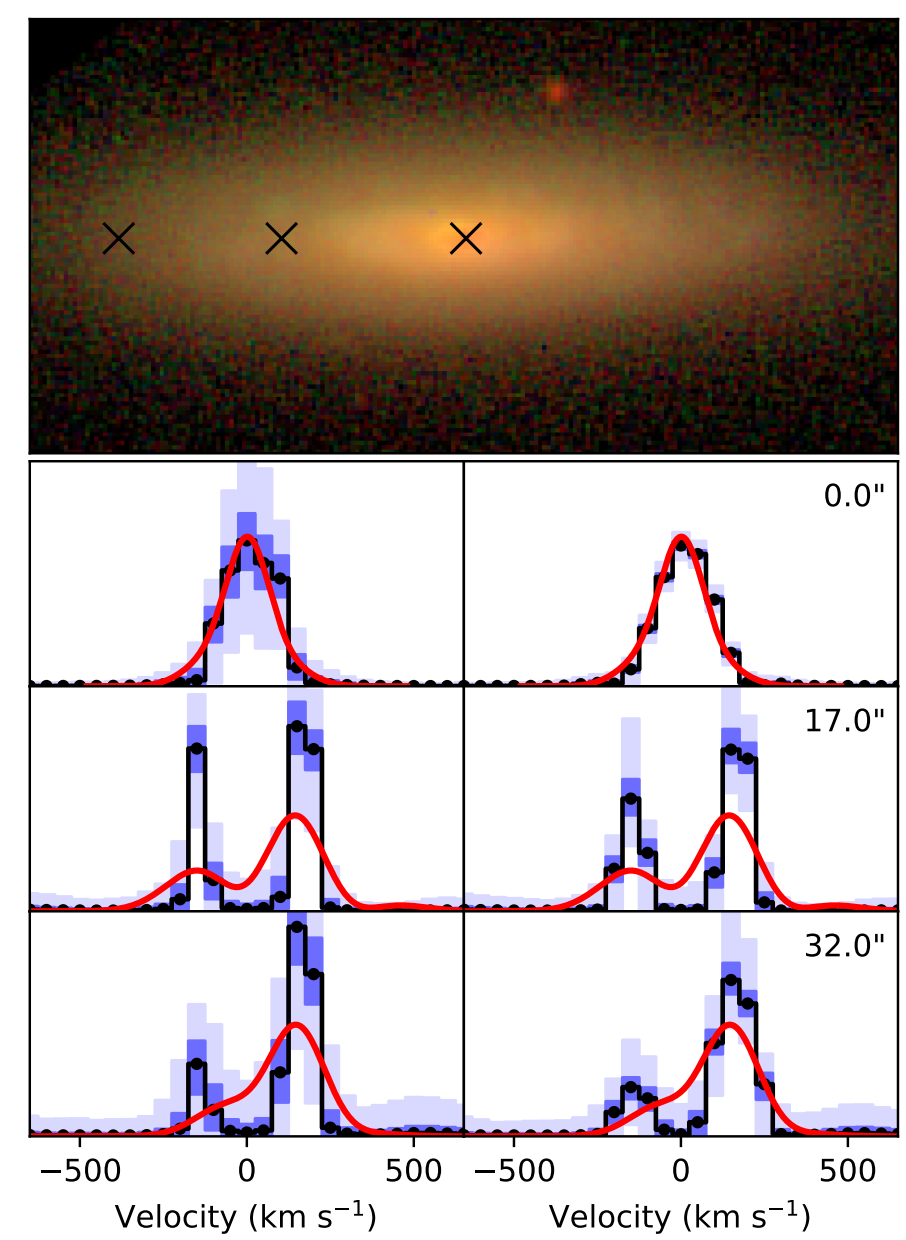

Fig. 6. BAYES-LOSVD extraction for IC 0719. Top panel: Sloan Digital Sky Survey colour image based on the $g, r, i$ filters. The image has been rotated so that the major axis is parallel to the abscissae. Bottom panels: LOSVDs extracted at different locations along the major axis of the galaxy (as indicated with black crosses on the image). In the left column, without regularisation, and in the right one with an order 2 auto-regressive prior. Colours are the same as in Fig. 2. Red lines correspond to the best Gauss-Hermite LOSVD extracted with pPXF (see Sect. 5.2 for details).

the non-parametric approach goes effectively to zero. It is worth highlighting the level of complexity of the recovered LOSVDs despite the smooth morphological appearance. The same applies to NGC 4550, as we discuss in Sect. 5.3, and emphasises the need for the non-parametric LOSVD extraction in galaxies. This topic is gaining attention in the literature, and non-parametric LOSVDs are now being routinely included in the dynamical modelling of early-type galaxies (e.g., Mehrgan et al. 2019; Neureiter et al. 2021).

Another interesting aspect to explore in this galaxy is the non-parametric extraction of the LOSVD in wavelength regions sensitive to different stellar populations. This is actually possible with MUSE data, and it will be a subject of analysis in Rubino et al. (in prep.).

\subsection{NGC 4550}

The last case we analysed was NGC 4550, another classical showcase galaxy with prominent double-peaked LOSVD profiles. We extracted LOSVDs from SAURON spectra (Emsellem et al. 2004). We performed our calculations in the wavelength range between $4800-5300 \AA$ and at a $S / N=150$ per spectral pixel. We analysed the results along the major axis of the galaxy at three of the locations presented in Rix et al. (1992). Since their data were not in electronic form, we digitised them using the WebPlotDigitiser ${ }^{15}$ tool (Marin et al. 2017) and then fitted the LOSVDs with double-Gaussian profiles for best reproduction. Figure 7 (top panel) shows the $F 555 W / F 814 W$ colour image from the Hubble Space Telescope. Remaining panels show our recovered LOSVDs at the three positions along the major axis, as indicated. Each row corresponds to a location, while each column uses different priors for the LOSVD extraction: no regularisation, auto-regressive (order 2), and auto-regressive (order 1). The red lines show the results of Rix et al. (1992).

The first thing to notice is the excellent agreement between our LOSVDs and those of Rix et al. (1992) when no regularisation is used. The complexity of the LOSVDs increases as we move away from the centre of the galaxy, as they become doublepeaked from 7.6". The results of Rix et al. (1992) are well within our $16 \%-84 \%$ confidence intervals (dark blue shaded region) despite the different sampling in velocity.

As opposed to IC 0719 , the situation is drastically different when regularisation is applied, however. Our auto-regressive (order 2) solutions are not capable of capturing the doublepeaked nature of the LOSVDs at larger distances from the centre. We investigated the source for this discrepancy and concluded that it is related to the intrinsic difference in velocity between the two peaks and the velocity sampling used to extract the LOSVDs. In other words, the level of correlation between velocity bins imposed by this prior is too strong and smooths the solution too much. In order to check this, we extracted the LOSVDs with an auto-regressive (order 1) prior, but we sampled the LOSVD in steps of $30 \mathrm{~km} \mathrm{~s}^{-1}$ instead of the $60 \mathrm{~km} \mathrm{~s}^{-1}$ used in all the other extractions. This is shown in the right-most column. It clearly shows that the two peaks can be recovered with a less stringent prior and finer sampling in velocity.

Based on these findings, we warn the reader that it is necessary to understand the implications of using regularisation in their analysis. We therefore recommend potential users to perform non-regularised fits on their data and carefully consider the velocity sampling to be used in the LOSVD extraction.

\section{Summary and conclusions}

The advent of very high quality data from many integral-field spectrographs and surveys has opened the possibility of efficiently extracting LOSVDs from galaxies. At the same time, great progress in computer performance, algorithms, and mathematical methods make it possible to handle large datasets. Inspired by the work of SW94, we developed a Bayesian inference approach to the LOSVD extraction from spectra. The code improves on SW94 in three main areas: (1) the MCMC sampling strategy; (2) the possibility of different forms of regularisation for the LOSVD; and (3) template optimisation based on PCA components. Our tests on mock data indicate that LOSVD recovery is accurate for spectra with $S / N>50$ with as few as five PCA templates. Regularised solutions provide less uncertain LOSVDs, but it is at the expense of biased solutions for low S/Ns. We also successfully applied our approach to MUSE and SAURON data, displaying many interesting features and warning

\footnotetext{
$\overline{15}$ https://automeris.io/WebPlotDigitizer
} 

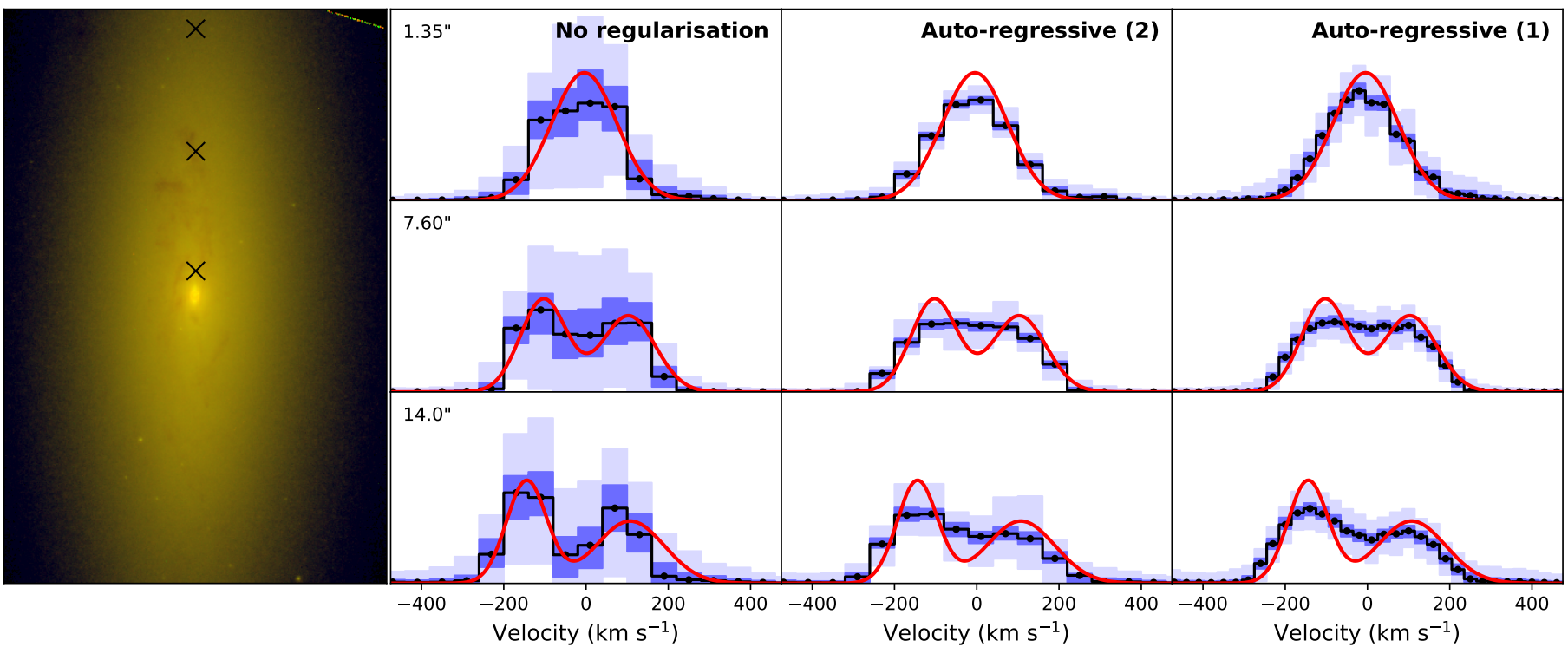

Fig. 7. BAYES-LOSVD extraction for NGC 4550. Left panel: Hubble Space Telescope colour image based on the $F 555 W$ and $F 814 W$ filters. North is up and east to the left. Other panels: LOSVDs extracted at several locations along the major axis of the galaxy (as marked by the black crosses). Each row corresponds to a different location from the centre of the galaxy (in arcsec) as shown on the first column. Each column uses a different kind of regularisation, as indicated in the top row. Colours are the same as in Fig. 2. Red lines show Rix et al. (1992) LOSVDs at those locations.

the users to be careful with regularised solutions in some situations (see Sect. 5.3 for an example). The use of non-regularised solutions should therefore be preferred, as it provides non-biased solutions.

On the technical side, our implementation is very versatile and allows the possibility of extending its capabilities on different fronts (i.e. inclusion of read-in routines for data of new instruments, new Stan models with different kinds of regularisation, and/or addition of new template libraries). The code and documentation can be downloaded from the repository indicated in Sect. 4.

The complexity in the kinematics observed in IFU surveys (e.g., Krajnović et al. 2011), but also numerical simulations (e.g., Martig et al. 2014; Schulze et al. 2017; Walo-Martín et al. 2020), clearly indicates that a non-parametric approach is necessary to capture the great level of detail that current data offer. This has been shown for decades in early-type galaxies, but the potential is much greater in late-type spiral systems, which display much more complex structures. In this respect, recent and upcoming large-scale IFU facilities (e.g., VIRUSW, Fabricius et al. 2008; MEGARA, Gil de Paz et al. 2018 and WEAVE-LIFU, Dalton et al. 2018) operating at spectral resolutions above $R \geq 5000$ open the door to explore the details of the LOSVDs in low velocity dispersion regimes (e.g., galaxy discs, dwarf galaxies), in which it has been very hard to operate with current instrumentation. The non-parametric description of the LOSVDs will also have a big impact on the decomposition of galaxies into their kinematic/dynamical components. Current efforts rely heavily on the smooth LOSVDs provided by Gauss-Hermite parametrisations (e.g., Tabor et al. 2017; Coccato et al. 2018; Oh et al. 2020). There is thus a great potential to go beyond those (necessary) efforts to explore galaxy mass assembly.

Acknowledgements. We thank the referee Prasenjit Saha for very useful comments that have helped improving the manuscript. We are grateful to Prashin Jethwa, Ignacio Martín-Navarro, Marc Sarzi, Glenn van de Ven, and Eugene Vasiliev for many inspiring discussions over the course of this project. We are also indebted to Michela Rubino for her feedback and suggestions while testing earlier versions of the code, and Alireza Molaeinezhad for assisting in the preparation of the software package for Github. We thank Alessandro Pizzella for letting us use the IC 0719 MUSE datacube in our analysis. J. F.-B. thanks Andrés Asensio Ramos for introducing him to the world of bayesian inference methods and Stan in particular. We thank Michele Cappellari for letting us include some of his python functions in our software package. NGC 4371 results are based on observations collected at the European Southern Observatory under ESO programme 060.A-9313(A). Software acknowledgements. Our code uses Astropy (https://www . astropy.org/) a community-developed core Python package for Astronomy (Astropy Collaboration 2013, 2018), as well as NumPy (https: //numpy.org/) (Oliphant 2006), SciPy (https://scipy.org/) (Jones et al. 2001) and Matplotlib (https://matplotlib.org/) (Hunter 2007). Funding and financial support acknowledgements. J. F.-B. acknowledges support through the RAVET project by the grant PID2019-107427GB-C32 from the Spanish Ministry of Science, Innovation and Universities (MCIU), and through the IAC project TRACES which is partially supported through the state budget and the regional budget of the Consejería de Economía, Industria, Comercio y Conocimiento of the Canary Islands Autonomous Community.

\section{References}

Asensio Ramos, A., de la Cruz Rodríguez, J., Martínez González, M. J. \& Socas-Navarro, H. 2017, A\&A, 599, A133

Astropy Collaboration (Robitaille, T. P., et al.) 2013, A\&A, 558, A33

Astropy Collaboration (Price-Whelan, A. M., et al.) 2018, AJ, 156, 123

Bender, R. 1990, A\&A, 229, 441

Betancourt, M. 2012, in AIP Conf. Ser., eds. P. Goyal, A. Giffin, K. H. Knuth, \& E. Vrscay, 1443, 157

Blanton, M. R., \& Roweis, S. 2007, AJ, 133, 734

Bruzual, G., \& Charlot, S. 2003, MNRAS, 344, 1000

Buchner, J. 2016, Stat. Comput., 26, 383

Cappellari, M. 2017, MNRAS, 466, 798

Cappellari, M., \& Copin, Y. 2003, MNRAS, 342, 345

Cappellari, M., \& Emsellem, E. 2004, PASP, 116, 138

Carpenter, B., Gelman, A., Hoffman, M., et al. 2017, J. Stat. Softw. Artic., 76, 1

Chen, Y.-M., Kauffmann, G., Tremonti, C. A., et al. 2012, MNRAS, 421, 314

Chilingarian, I., Prugniel, P., Sil'Chenko, O., \& Koleva, M. 2007, in Stellar

Populations as Building Blocks of Galaxies, eds. A. Vazdekis, \& R. Peletier, IAU Symp., 241, 175

Coccato, L., Morelli, L., Pizzella, A., et al. 2013, A\&A, 549, A3

Coccato, L., Fabricius, M. H., Saglia, R. P., et al. 2018, MNRAS, 477, 1958

Coelho, P., Barbuy, B., Meléndez, J., Schiavon, R. P., \& Castilho, B. V. 2005, A\&A, 443, 735 
Dalton, G., Trager, S., Abrams, D. C., et al. 2018, in Ground-based and Airborne Instrumentation for Astronomy VII, SPIE Conf. Ser., 10702, 107021B

Deason, A. J., Belokurov, V., \& Evans, N. W. 2011, MNRAS, 411, 1480

Debattista, V. P., Ness, M., Earp, S. W. F., \& Cole, D. R. 2015, ApJ, 812, L16

de Bruyne, V., Vauterin, P., de Rijcke, S., \& Dejonghe, H. 2003, MNRAS, 339, 215

Du, H., Mao, S., Athanassoula, E., Shen, J., \& Pietrukowicz, P. 2020, MNRAS 498, 5629

Duane, S., Kennedy, A. D., Pendleton, B. J., \& Roweth, D. 1987, Phys. Lett. B, 195,216

Dullo, B. T., Bouquin, A. Y. K., Gil de Paz, A., Knapen, J. H., \& Gorgas, J. 2020, ApJ, 898, 83

Emsellem, E., Cappellari, M., Peletier, R. F., et al. 2004, MNRAS, 352, 721

Emsellem, E., Cappellari, M., Krajnović, D., et al. 2011, MNRAS, 414, 888

Erwin, P., Vega Beltrán, J. C., \& Beckman, J. E. 2001, in The Central Kiloparsec of Starbursts and AGN: The La Palma Connection, eds. J. H. Knapen, J. E. Beckman, I. Shlosman, \& T. J. Mahoney, ASP Conf. Ser., 249, 171

Fabricius, M. H., Barnes, S., Bender, R., et al. 2008, in Ground-based and Airborne Instrumentation for Astronomy II, SPIE Conf. Ser., 7014, 701473

Fabricius, M. H., Coccato, L., Bender, R., et al. 2014, MNRAS, 441, 2212

Falcón-Barroso, J., Balcells, M., Peletier, R. F., \& Vazdekis, A. 2003, A\&A, 405 455

Falcón-Barroso, J., Lyubenova, M., van de Ven, G., et al. 2017, A\&A, 597, A48

Foreman-Mackey, D., Hogg, D. W., Lang, D., \& Goodman, J. 2013, PASP, 125, 306

Franx, M., \& Illingworth, G. D. 1988, ApJ, 327, L55

Gadotti, D. A., Seidel, M. K., Sánchez-Blázquez, P., et al. 2015, A\&A, 584, A90

Gadotti, D. A., Sánchez-Blázquez, P., Falcón-Barroso, J., et al. 2019, MNRAS, 482,506

Gebhardt, K., Richstone, D., Kormendy, J., et al. 2000, AJ, 119, 1157

Geman, S., \& Geman, D. 1984, IEEE Transactions on Pattern Analysis and Machine Intelligence, PAMI-6, 721

Gil de Paz, A., Carrasco, E., Gallego, J., et al. 2018, in Ground-based and Airborne Instrumentation for Astronomy VII, SPIE Conf. Ser., 10702 1070217

Gonneau, A., Lyubenova, M., Lançon, A., et al. 2020, A\&A, 634, A133

González-García, A. C., Balcells, M., \& Olshevsky, V. S. 2006, MNRAS, 372, L78

Halliday, C., Davies, R. L., Kuntschner, H., et al. 2001, MNRAS, 326, 473

Hastings, W. K. 1970, Biometrika, 57, 97

Hoffman, M. D., \& Gelman, A. 2014, J. Mach. Learn. Res., 15, 1593

Hunter, J. D. 2007, Comput. Sci. Eng., 9, 90

Jesseit, R., Naab, T., Peletier, R. F., \& Burkert, A. 2007, MNRAS, 376, 997

Jones, E., Oliphant, T., Peterson, P., et al. 2001, SciPy: Open Source Scientific tools for Python, http: //www.scipy.org/

Jore, K. P., Broeils, A. H., \& Haynes, M. P. 1996, AJ, 112, 438

Katkov, I., Chilingarian, I., Sil'chenko, O., Zasov, A., \& Afanasiev, V. 2011, Balt Astron., 20, 453

Katkov, I. Y., Sil'chenko, O. K., \& Afanasiev, V. L. 2013, ApJ, 769, 105

Kelson, D. D., Illingworth, G. D., van Dokkum, P. G., \& Franx, M. 2000, ApJ, 531,159

Krajnović, D., Emsellem, E., Cappellari, M., et al. 2011, MNRAS, 414, 2923

Kruschke, J. 2014, Doing Bayesian Data Analysis: A Tutorial with R, JAGS, and Stan (Academic Press)

Kuijken, K., \& Merrifield, M. R. 1993, MNRAS, 264, 712

Kuijken, K., Fisher, D., \& Merrifield, M. R. 1996, MNRAS, 283, 543

Kunder, A., Koch, A., Rich, R. M., et al. 2012, AJ, 143, 57

Lamperti, I., Saintonge, A., De Looze, I., et al. 2019, MNRAS, 489, 4389

Lawson, C. L., \& Hanson, R. J. 1974, Prentice-Hall Series in Automatic Computation (Englewood Cliffs: Prentice-Hall)
Li, C., Wang, T.-G., Zhou, H.-Y., Dong, X.-B., \& Cheng, F.-Z. 2005, AJ, 129, 669

Liu, Q., \& Wang, D. 2016, ArXiv e-prints [arXiv:1608.04471]

Lu, H., Zhou, H., Wang, J., et al. 2006, AJ, 131, 790

Maraston, C., Hill, L., Thomas, D., et al. 2020, MNRAS, 496, 2962

Marin, F., Rohatgi, A., \& Charlot, S. 2017, in SF2A-2017: Proceedings of the Annual Meeting of the French Society of Astronomy and Astrophysics, eds. C. Reylé, P. Di Matteo, F. Herpin, et al., 113

Martig, M., Minchev, I., \& Flynn, C. 2014, MNRAS, 443, 2452

Mehrgan, K., Thomas, J., Saglia, R., et al. 2019, ApJ, 887, 195

Merritt, D. 1997, AJ, 114, 228

Metropolis, N., Rosenbluth, A. W., Rosenbluth, M. N., Teller, A. H., \& Teller, E. 1953, J. Chem. Phys., 21, 1087

Moré, J., Garbow, B., \& Hillstrom, K. 2001, User Guide for MINPACK1. Argonne National Laboratory Argonne, IL, http://cds.cern.ch/ record/126569

Ness, M., Freeman, K., Athanassoula, E., et al. 2013, MNRAS, 432, 2092

Neureiter, B., Thomas, J., Saglia, R., et al. 2021, MNRAS, 500, 1437

Nolan, L. A., Harva, M. O., Kabán, A., \& Raychaudhury, S. 2006, MNRAS, 366, 321

Norris, J. 1986, ApJS, 61, 667

Ocvirk, P., Pichon, C., Lançon, A., \& Thiébaut, E. 2006, MNRAS, 365, 74

Oh, S., Colless, M., Barsanti, S., et al. 2020, MNRAS, 495, 4638

Oliphant, T. E. 2006, A Guide to NumPy (USA: Trelgol Publishing), 1

Parviainen, H. 2018, Bayesian Methods for Exoplanet Science (Springer International Publishing AG), 149

Pinkney, J., Gebhardt, K., Bender, R., et al. 2003, ApJ, 596, 903

Pizzella, A., Morelli, L., Coccato, L., et al. 2018, A\&A, 616, A22

Press, W. H., Teukolsky, S. A., Vettering, W. T., \& Flannery, B. P. 2003, Eur. J. Phys., 24, 329

Prugniel, P., Soubiran, C., Koleva, M., \& Le Borgne, D. 2007, ArXiv e-prints [arXiv:astro-ph/0703658]

Richards, J. W., Freeman, P. E., Lee, A. B., \& Schafer, C. M. 2009, ApJ, 691, 32

Rix, H.-W., Franx, M., Fisher, D., \& Illingworth, G. 1992, ApJ, 400, L5

Ronen, S., Aragon-Salamanca, A., \& Lahav, O. 1999, MNRAS, 303, 284

Saha, P., \& Williams, T. B. 1994, AJ, 107, 1295

Salvatier, J., Wiecki, T. V., \& Fonnesbeck, C. 2016, PeerJ Comput. Sci., 2, 55

Sánchez Almeida, J., \& Allende Prieto, C. 2013, ApJ, 763, 50

Sánchez-Blázquez, P., Peletier, R. F., Jiménez-Vicente, J., et al. 2006, MNRAS, 371,703

Sargent, W. L. W., Schechter, P. L., Boksenberg, A., \& Shortridge, K. 1977, ApJ, 212,326

Schulze, F., Remus, R.-S., \& Dolag, K. 2017, Galaxies, 5, 41

Simkin, S. M. 1974, A\&A, 31, 129

Statler, T. 1995, AJ, 109, 1371

Stenning, D. C., Wagner-Kaiser, R., Robinson, E., et al. 2016, ApJ, 826, 41

Tabor, M., Merrifield, M., Aragón-Salamanca, A., et al. 2017, MNRAS, 466, 2024

Tolstoy, E., Irwin, M. J., Helmi, A., et al. 2004, ApJ, 617, L119

Tonry, J., \& Davis, M. 1979, AJ, 84, 1511

Valdes, F., Gupta, R., Rose, J. A., Singh, H. P., \& Bell, D. J. 2004, ApJS, 152, 251

van de Sande, J., Bland-Hawthorn, J., Fogarty, L. M. R., et al. 2017, ApJ, 835, 104

van der Marel, R. P., \& Franx, M. 1993, ApJ, 407, 525

Vazdekis, A., Sánchez-Blázquez, P., Falcón-Barroso, J., et al. 2010, MNRAS, 404, 1639

Walo-Martín, D., Falcón-Barroso, J., Dalla Vecchia, C., Pérez, I., \& Negri, A. 2020, MNRAS, 494, 5652

Zoccali, M., Vasquez, S., Gonzalez, O. A., et al. 2017, A\&A, 599, A12 


\section{Appendix A: S/N trends}

In this appendix, we present the equivalent figures to Fig. 4 for different $\mathrm{S} / \mathrm{Ns}$. It is evident that the LOSVD recovery worsens as $\mathrm{S} / \mathrm{N}$ decreases. It is interesting to see that not all the features are captured well, even at $S / N=100$ (e.g., small bump on the positive wing of the case in the bottom row in all figures).

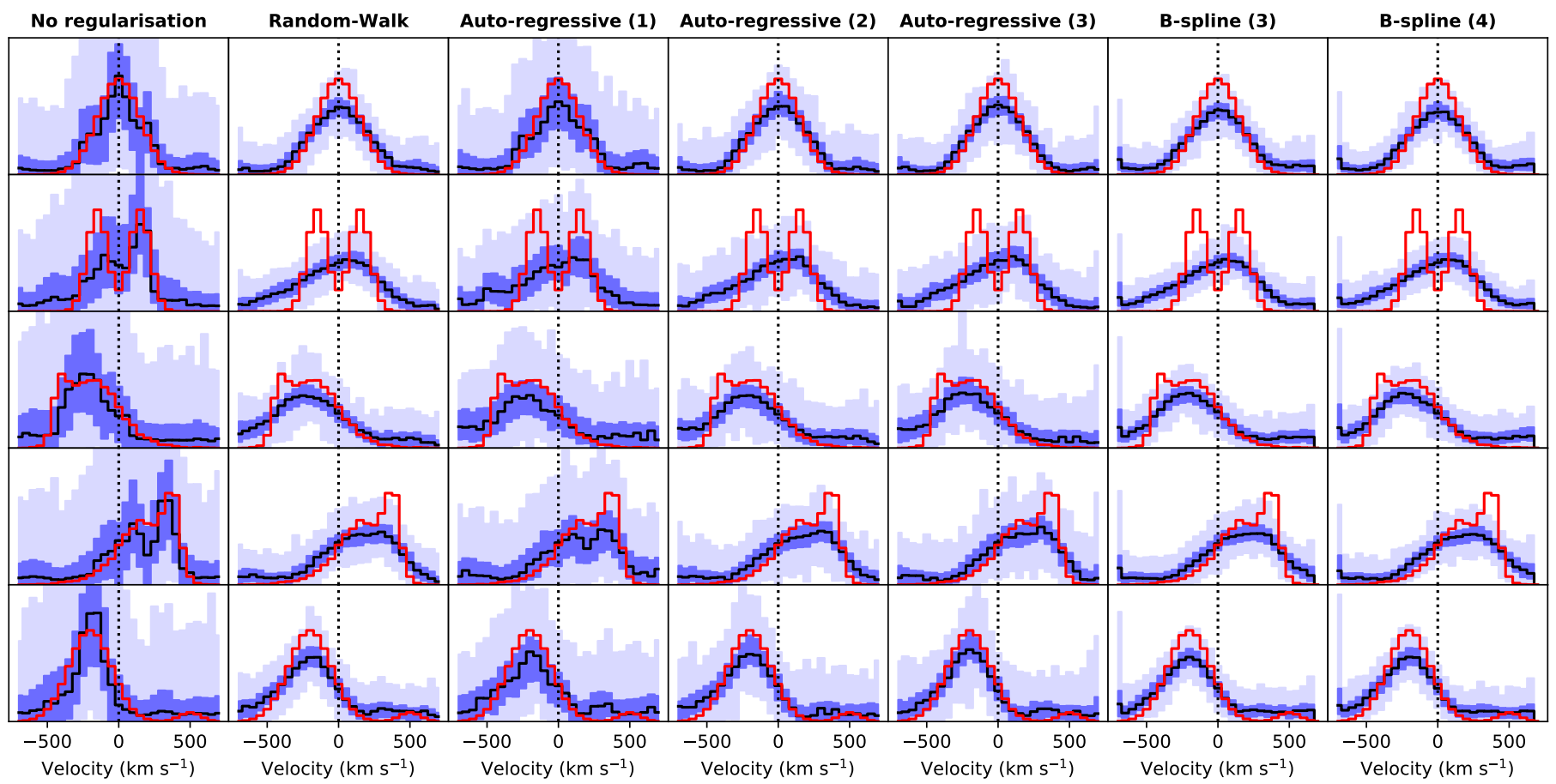

Fig. A.1. Same as Fig. 4, but for $S / N=10$.
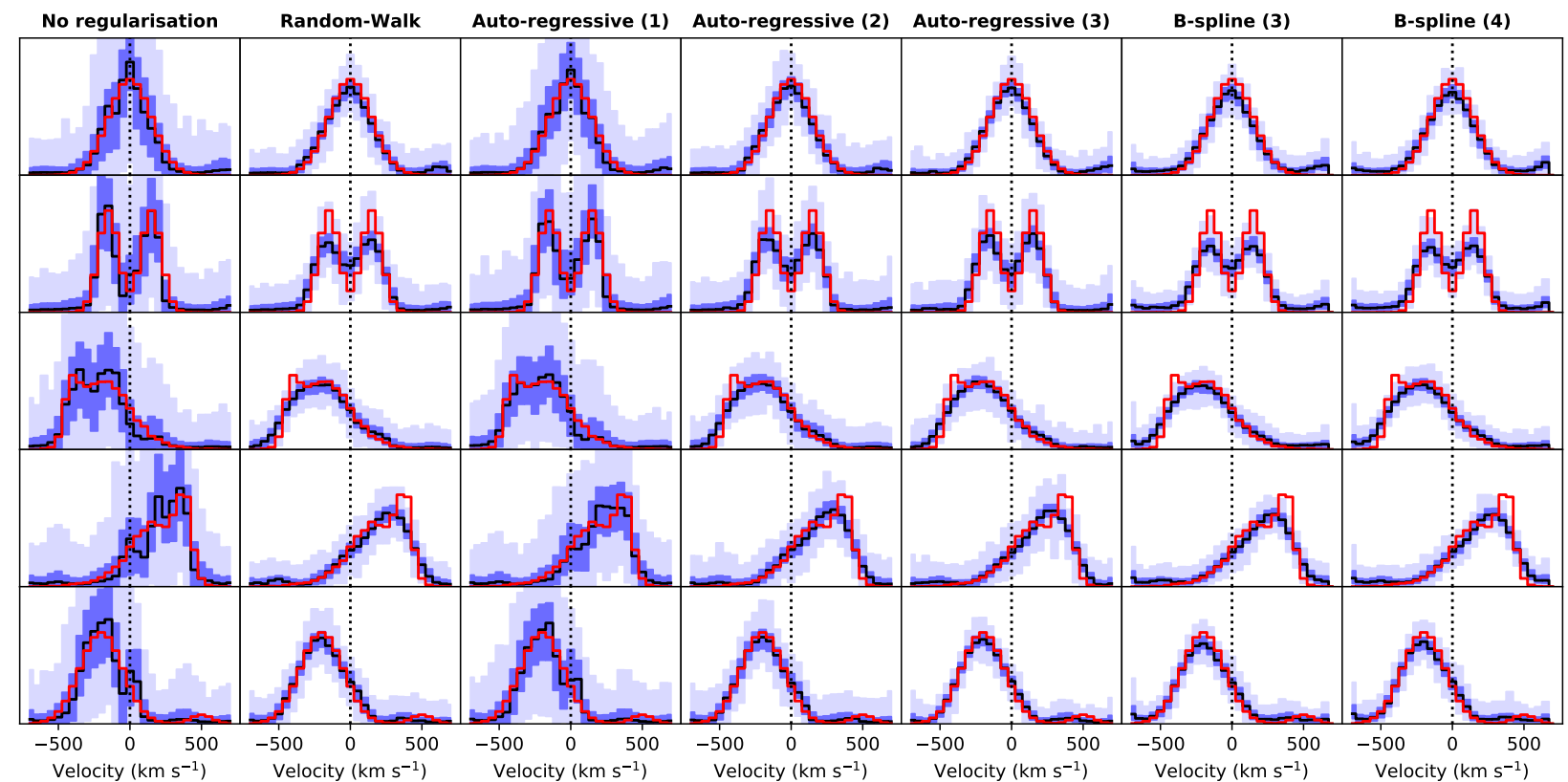

Fig. A.2. Same as Fig. 4, but for $S / N=25$. 


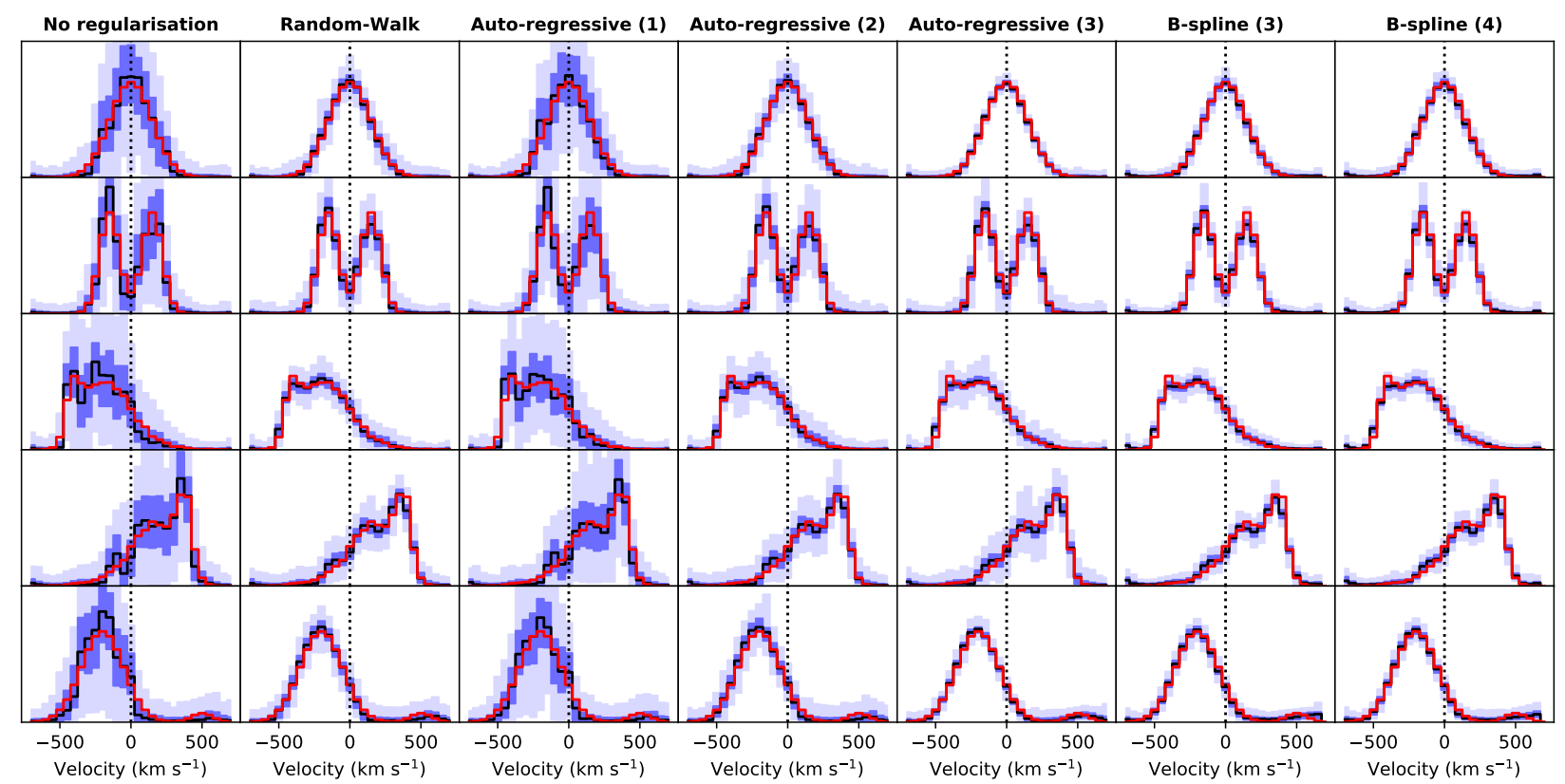

Fig. A.3. Same as Fig. 4, but for $S / N=100$.

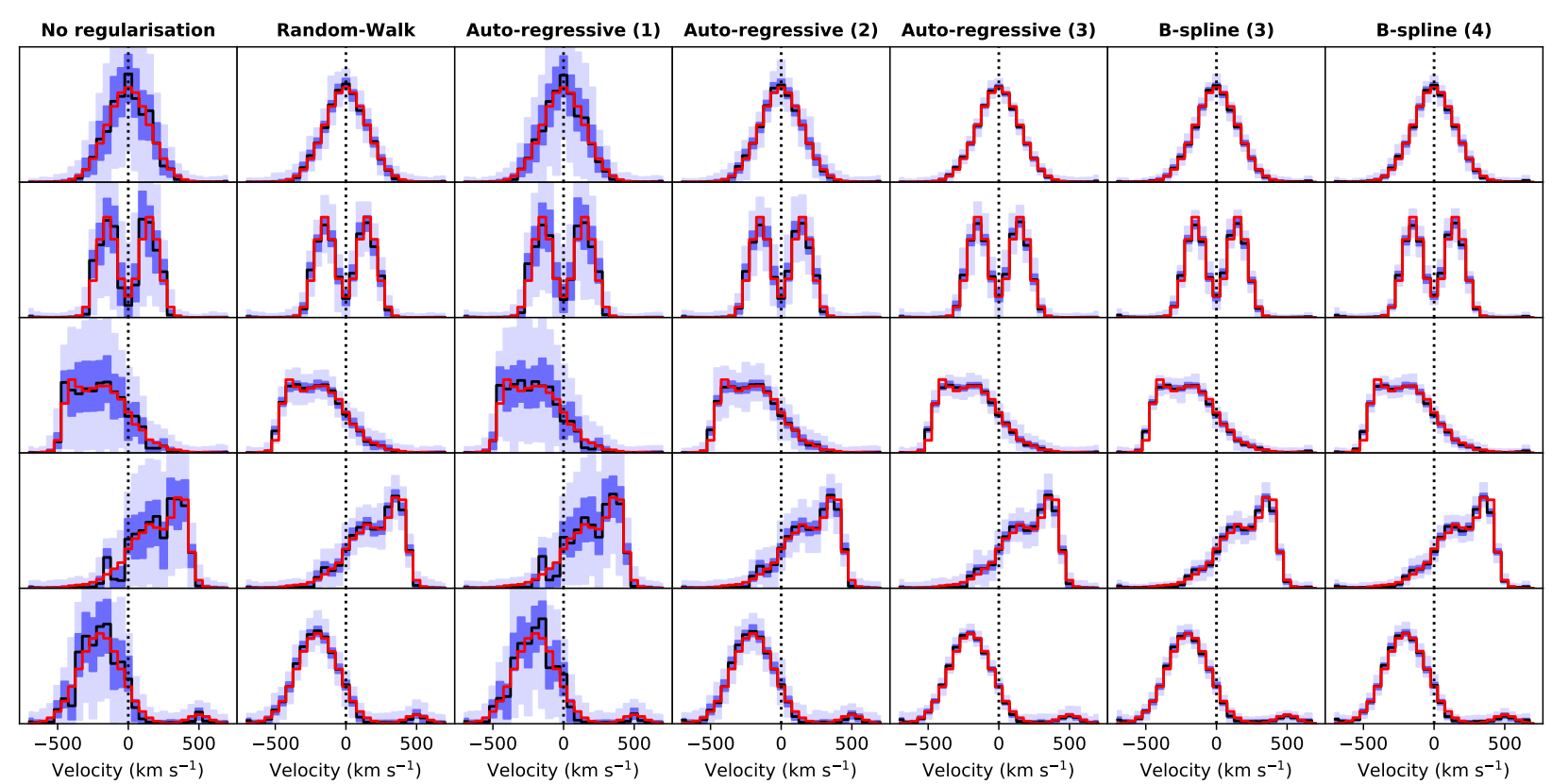

Fig. A.4. Same as Fig. 4, but for $S / N=200$. 